\title{
Konsequenzen, die der Autor Kafka zog, oder: Das physikalische, elektromagnetische Experiment als Inspirationsquelle - eine Beweisführung aus dem Geiste der „Dritten Kultur"
}

Man lebte, wie ausgeführt, damals bereits in einer Gesellschaft, in der der soziale Bahnwechsel (vornehmlich in Form der Judenassimilation) gang und gäbe geworden war. Eine solche Gesellschaft ist nicht mehr mechanisch zu nennen. Sondern muss als dynamisch gelten, auch in diesem Sinn als ,liberal“ im Sinne des politikwissenschaftlich-historischen Terminus'. Die elektromagnetische Feldtheorie als Bild/Metapher für die sozialen Verhältnisse gehört ausschließlich der liberalen Gesellschaft an, wie sie im 19. Jahrhundert sich konstituiert hatte. Ein Freiheitsgewinn von enormer Dimension. Aber andererseits eben doch auch eine Auflösung der Ordnung. Deshalb von Denkern wie Berkeley oder Dilthey panisch gefürchtet, Stoff dann auch für Apologeten des Ständestaats, vom ,Verlust der Mitte"-Sedlmayr bis hin zu, auf höherem Niveau, Ortega y Gasset. Alle diese neuen Denkmodelle formten die „moderne Welt“ in Franz Kafkas Lebens- und Schreibzeit, Botho Strau $\beta$ hat darüber intensiv nachgedacht in seiner jüngeren Novellenproduktion, wie immer ohne Kafkas Namen zu erwähnen. Dessen besprochener Jugendroman kannte dieses „Kafkaeske“ noch nicht. In ihm wurde das Unheimliche sogar intertextuell aufgelöst. Denn des Romantikers Heinrich von Kleist ,unheimliche“ Kampf-Verstrickung der ,elektrisch aufgeladenen“, ihrerseits wie aus der Bahn geratene Elektroden agierenden Kampfmaschinen Achill und Penthesilea, diese fatale Kampfszene wird vom frühen, noch optimistischen Kafka überführt in Klaras eher komisch anmutenden JiuJitsu-Kampf. Wurde derart angepasst der restlos erleuchteten, gnadenlos ausgeleuchteten Welt des neuesten New York. Diese Welt ist bereits durchgehend elektrifiziert und liegt nicht mehr im Licht des Mondscheins, sondern in dem

B. Neumann, Umrisse einer Dritten Kultur im interdisziplinären Zusammenspiel zwischen Literatur und Naturwissenschaft, ELECTRISCHER PROMETHEUS. Umrisse einer Dritten Kultur im interdisziplinären Zusammenspiel zwischen Literatur und Naturwissenschaft, https://doi.org/10.1007/978-3-662-63204-8_20 
von Autoscheinwerfern oder elektrischen Büroleuchten. Gleicht einem hypermodernen Versandhandel nach dem Muster bereits von Amazon:

„Es war daher ein Geschäft, welches in einem Käufe, Lagerungen, Transporte und Verkäufe riesenhaften Umfangs umfasste und ganz genaue unaufhörliche telefonische und telegraphische Verbindungen mit den Kunden unterhalten musste ... man sah dort im sprühenden elektrischen Licht einen Angestellten, ... den Kopf eingespannt in ein Stahlband, das ihm die Hörmuscheln an die Ohren drückte."1

Das elektromagnetische Feld geriet hierin zur alles bestimmenden Größe, und Einsteins Konzeption der Lichtgeschwindigkeit zu einem neuen Absoluten. Die Lichtgeschwindigkeit zu erreichen konnte nun metaphorisch für den Wunschzustand endlich geglückter, unwiderruflicher Assimilation stehen. Also wundersam aufgehobene „Aporien der Assimilation“ - die es aber nicht gibt. Weder kann man die Lichtgeschwindigkeit überschreiten, noch die Assimilation zu umfassend geglücktem Erfolg führen. Beide Phänomene erweisen sich als prinzipiell unerreichbar, sie sind eben dadurch bestimmt, verfügen in der Unerreichbarkeit über ihre eigentliche Substanz. Wir leben zudem unsere Alltage in einer Welt, in der Newtons Mechanik weiterhin gilt, zusammen mit dem „Bildungs“"- oder „Realistischen Roman“, und wo ständig rekonstruierte, wieder ganz traditionelle, wenn man so will: „Mechanische“ Erzählformen erneut das Sagen besitzen. Der „Nouveau Roman“ beispielsweise ist längst tot - aber Franz Kafkas (gemessen an diesem traditionelles) Erzählen, es lebt. Denn allein dem Prager gelang es, jene „,narrative Relativitätstheorie“ in erzählerische Praxis zu transformieren, die Axel Gellhaus proklamiert und entwickelt hat und deren einzelne Entwicklungsschritte hier demonstriert werden. Folgerichtig, dass dies alles in Prag geschah, der immer schon magischen Stadt, wo man nicht erst seit der Renaissance auf die allumfassende Weltformel aus war. Hier entstand Kafkas erster durchgehend „,kafkaesker“ Text: Die Geschichte vom Junggesellen Blumfeld, niedergeschrieben im Jahr 1915, eine Keimzelle des späteren Schlosses, letzteres eine Erkenntnis, die die Kafka-Forschung weltweit anerkennt.

Man muss es also „den Buben sagen“ (und warten, was dann geschieht). Drei Jahre nach der Einstein-Begegnung jedenfalls zog Kafka die poetologischen und schreibpraktischen Konsequenzen. Im Jahr 1915, genauer: Zwischen dem 8. Februar und März/April desselben Jahres (eine Ehe mit Felice kommt eigentlich seit 1912 nicht mehr in Frage; die sublimiert-erotische Verlockung des

\footnotetext{
${ }^{1}$ Franz Kafka, Der Verschollene, in: Taschenbuchausgabe in sieben Bänden, hrsg. v. Max Brod, Frankfurt/Main 2004, Bd. I., S. 54 (vgl. Anmerkung 38).
} 
Nacht-Schreibens hat bereits endgültig über die hergebrachte Erotik gesiegt), schreibt Kafka seine Blumfeld-Junggesellengeschichte nieder. Eine Erzählung, im Tagebuch aufgezeichnet, die ganz unverkennbar bereits einen Erzählkern für den späteren Schloss-Roman bildete. Die beiden geheimnisvoll „kafkaesken“ Bälle, die danach in hüpfende „Gehilfen“ sich verwandeln, treten hier zuerst in ihr literarisches Leben - das sie danach noch bis ins Schloss führen wird. 1915 geschieht dies, direkt nachdem Kafka in Bodenbach, im Januar bereits und auch in nächtlichem Zusammensein, erfahren hatte, dass Felice als Ehepartnerin mit Sicherheit nicht infrage komme. Nun war Kafka selbst zu Blumfeld geworden, - zu einem Nachtschreiber, der seit dem Urteil wusste, dass Nachtschreiben lustvoller sein kannAtomkerns als aller nächtlicher Beischlaf, jedenfalls wenn die Partnerin Felice hieß. Und: Kafka war damals auf Zimmersuche, richtete sich buchstäblich ganz neu ein, erfuhr die Einsamkeit des Junggesellen, dem keine freundliche Ehefrau die Tür öffnete und den kein freudig bellender Hund erwartete. Stattdessen: Verwirrend elektrisch hüpfende Bälle, die sich an keine feste Bahn mehr halten. Moderne Atomphysik anstelle von Newton/Leibniz' noch „mechanisch“ zusammengehaltenem Kosmos (und einem nach dessen harmonischen Gesetzen ausgerichteten Familienleben). Elektromagnetisch aufgeladene, irrlichternde Kugeln statt eines treu blickenden, zuweilen bellenden, zuweilen heulenden Hundes. Von einer „Hundegeschichte“ sprachen Kafkas erste Aufzeichnungen zum Blumfeld.

Dabei wusste der moderne Autor Franz Kafka, was er tat. Denn der Prager hat die Blumfeld-Geschichte, als diese im Januar 1915 in eine Schreibkrise geraten war, kommentiert wie folgt: „... auf einer Sandbank ein noch knapp atmender Fisch. Ich schreibe „Bouvart und Pécuchet“ sehr frühzeitig.“2 Was bedeutet: Kafka ließ sich von Flauberts nachgelassenem, Fragment gebliebenem Roman nicht nur inspirieren, was die Personenkonstellation und -zeichnung betraf. Sondern zwei verwechselbare, automatenhafte Gestalten werden nun vorgestellt, ganz wie bei dem Franzosen die titelgebenden „Helden“; bei Kafka erst in Blumfelds Wohnung, danach in seinem Büro - und schließlich im Schloss-Roman von 1922. Die Schöpfung zweier Roman-Protagonisten aus den Utensilien und Verläufen eines physikalischen Experiments ereignet sich hier! Mehr noch: Franz Kafka beruft sich auf einen Roman, der seinerseits auch ein (gelehrtes, wie immer parodisches) Kompendium von Wissenschaftsparadigmen darstellt. Flauberts nachgelassener Roman ist ja eine eingehende Thematisierung

${ }^{2}$ Franz Kafka, Tagebücher, 9. II. 1915. 
des epistemologischen Bruchs zwischen dem 18. und 19. Jahrhundert, wofür Kindlers Neues Literatur Lexikon Foucaults Wissenschafts-Systematik samt „Archiv“ und „Paradigmawechsel“ ins Feld führt. ${ }^{3}$ Mithin berief Kafka sich hier auf einen in der Literatur thematisierten Paradigmawechsel innerhalb der Wissenschaft, wie ihn Flauberts Text (in diesem ging es vor allem um die Ablösung des Positivismus) dargestellt hatte. Damit liegt hier sogar eine Meta-Reflektion des intertextuell Praktizierten vor: Es geht um des Pragers Eigeneinordnung in die Nachfolge Flauberts, wo dieser ein enorm ehrgeiziges, weil von Wissenschaftsreflexion gesättigtes Werk verfasst hatte (und in dem die Begründung für Michel Houellebecqs Diktum, dass man keine gute Literatur ohne Kenntnis der je aktuellen naturwissenschaftlichen Entwicklungen schreiben könne, als eine - hier auch für Kafka geltende - französische Tradition erscheinen könnte)? Und: Hans Magnus Enzensbergers Essay zum Thema „Dritte Kultur“ wird sich eben auf Flauberts genannten Roman berufen, wobei es überflüssig erscheint zu erwähnen, dass Kafka und Enzensberger völlig unabhängig voneinander auf den Franzosen als Eideshelfer verfallen sind. Davon gleich noch mehr. Weiterhin: Die erst physikalischen, dann kafkaesken Bälle werden von Kafka noch im Jahr 1915 in zwei „Gehilfen“ transfiguriert. Mithin wird Physik in Gesellschaftliches erzählerisch verwandelt. Es geschieht der entscheidende Schritt in Richtung auf die spätere Konstellation: Erst Blumfeld, dann K. mit ihren elementarteilchenhaften „Gehilfen“, die im Schloss dann verwirrt/verwirrend ihre Bahn wechseln, sich zusammenklumpen, überraschende Sprünge vollziehen etc. Wir befinden uns plötzlich bereits in der Welt des Atoms, fiktional vorweggenommen als Lizenz modernsten Schreibens. Dazu Hartmut Binder: „Die Bälle korrespondieren mit den beiden später eingeführten Praktikanten Blumfelds; dieses Motiv auch im Schloss. "4 Mithin ein Einschnitt, eine epochemachende Transformation in Kafkas Schreiben. Das zu erkennen ist entscheidend. Aber leider nicht auf den ersten Blick. Denn Hartmut Binder, immer noch der Häuptling aller Kafka-Rechercheure, ist eine Unterlassung unterlaufen. Will sagen: In Binders Kommentar zur Blumfeld-Geschichte wird noch nicht erwähnt, was Binder allerdings in seinem Handbuch dann ausführlich beschreiben wird: Dass nämlich Kafka bei der Konstruktion der kafkaesken Bälle ganz direkt auf den Physik-Unterricht an seiner Schule zurückgriffen hatte (die war keineswegs so

\footnotetext{
${ }^{3}$ Kindlers Neues Literatur Lexikon, hrsg. v. Walter Jens, München 1988, im Artikel zu Gustave Flauberts „Bouvart und Pécuchet“.

${ }^{4}$ Hartmut Binder, KafkaKommentar zu sämtlichen Erzählungen, München 1975, S. 192.
} 
verschnarcht, wie man sie heute gern auffasst). Doch existiert nicht nur diese eine benennbare, fraglos vorhandene, biographisch festmachbare Quelle für Kafkas „physikalische“ Inspiration. Sondern er und Brod trafen auf eben die gleiche physikalische Demonstration in Paris. Brod war damals im Bois de Boulogne, im westlichen Teil von Paris und noch vor Weltkriegsbeginn, der Zeuge beim déja-vu. Und er hat das aufgeschrieben: „Das elektrische Experiment mit den springenden Kügelchen, an das sich Blumfeld in der gleichnamigen Erzählung angesichts der beiden hüpfenden Bälle erinnert (B144), sah Kafka nicht im Gymnasium (jedenfalls nicht nur), sondern (auch, B.N.) während seines ParisAufenthalts im Jahr 1911 (M. Brod, 1912, S. 2)."5 Quod erat demonstrandum. Die Geburt des „Kafkaesken“ aus dem Geiste der neuesten Physik, und das keineswegs als Zangengeburt „,bloßer Spekulation“. Dieser Säugling schreit vielmehr ganz kräftig und fast schon melodisch-tonal.

${ }^{5}$ Hartmut Binder, Kafka-Handbuch, Stuttgart 1979, Bd. I, S. 193. 
Open Access Dieses Kapitel wird unter der Creative Commons Namensnennung 4.0 International Lizenz (http://creativecommons.org/licenses/by/4.0/deed.de) veröffentlicht, welche die Nutzung, Vervielfältigung, Bearbeitung, Verbreitung und Wiedergabe in jeglichem Medium und Format erlaubt, sofern Sie den/die ursprünglichen Autor(en) und die Quelle ordnungsgemäß nennen, einen Link zur Creative Commons Lizenz beifügen und angeben, ob Änderungen vorgenommen wurden.

Die in diesem Kapitel enthaltenen Bilder und sonstiges Drittmaterial unterliegen ebenfalls der genannten Creative Commons Lizenz, sofern sich aus der Abbildungslegende nichts anderes ergibt. Sofern das betreffende Material nicht unter der genannten Creative Commons Lizenz steht und die betreffende Handlung nicht nach gesetzlichen Vorschriften erlaubt ist, ist für die oben aufgeführten Weiterverwendungen des Materials die Einwilligung des jeweiligen Rechteinhabers einzuholen.

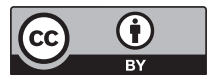

